\title{
Electrically Injected Twin Photon Emitting Lasers at Room Temperature
}

\author{
Claire Autebert ${ }^{1}$, Giorgio Maltese ${ }^{1}$, Yacine Halioua ${ }^{1}$, Fabien Boitier ${ }^{1,2}$, Aristide Lemaître ${ }^{3}$, \\ Maria Amanti ${ }^{1}$, Carlo Sirtori ${ }^{1}$ and Sara Ducci ${ }^{1, *}$ \\ 1 Laboratoire Matériaux et Phénomènes Quantiques, Université Paris Diderot, Sorbonne Paris Cité, \\ Paris 75205, France; claire.autebert@univ-Paris-diderot.fr (C.A.); \\ giorgio.maltese@univ-Paris-diderot.fr (G.M.); yacine.halioua@univ-Paris-diderot.fr (Y.H.); \\ fabien.boitier@nokia-bell-labs.com (F.B.); maria.amanti@univ-Paris-diderot.fr (M.A.); \\ carlo.sirtori@univ-Paris-diderot.fr (C.S.) \\ 2 Nokia Bell Labs, Route de Villejust, Nozay 91620, France \\ 3 Centre de Nanosciences et de Nanotechnologies, CNRS/Université Paris Sud, Marcoussis 91460, France; \\ aristide.lemaitre@lpn.cnrs.fr \\ * Correspondence: sara.ducci@univ-Paris-diderot.fr; Tel.: +33-1-5727-6225
}

Academic Editor: Stephan Reitzenstein

Received: 29 June 2016; Accepted: 8 August 2016; Published: 18 August 2016

\begin{abstract}
On-chip generation, manipulation and detection of nonclassical states of light are some of the major issues for quantum information technologies. In this context, the maturity and versatility of semiconductor platforms are important assets towards the realization of ultra-compact devices. In this paper we present our work on the design and study of an electrically injected AlGaAs photon pair source working at room temperature. The device is characterized through its performances as a function of temperature and injected current. Finally we discuss the impact of the device's properties on the generated quantum state. These results are very promising for the demonstration of electrically injected entangled photon sources at room temperature and let us envision the use of III-V semiconductors for a widespread diffusion of quantum communication technologies.
\end{abstract}

Keywords: entanglement production; semiconductor laser; integrated quantum optics

\section{Introduction}

Integrated quantum photonics is a very active field of quantum information science. In particular, the maturity of semiconductor technology offers a huge potential to build ultra-compact devices including generation, manipulation and detection of many quantum bits. In these last years, spectacular progress has been done on different material platforms, such as AlGaAs, silicon-on-insulator [1,2], silica-on-silicon [3,4], as well as on their hybrid integration, e.g., with superconducting detectors [5]. In this context, the direct band gap and electro-optics effect characterizing III-V semiconductors are important assets for the achievement of electrically injected, tunable, integrated quantum photonic devices. While quantum dots-based sources working at cryogenic temperatures have allowed us to reach unprecedented levels of brightness and to tune the degree of photon indistinguishability [6], spontaneous parametric down-conversion (SPDC) in $\mathrm{AlGaAs}$ has allowed us to produce entanglement on various degrees of freedom with devices working at room temperature and telecom wavelength (see Section 3).

Recently, the utilization of this platform has led to the demonstration of a completely integrated electrically injected device consisting of a quantum-well laser emitting photons at $780 \mathrm{~nm}$ that are converted into telecom-wavelength photon pairs by internal spontaneous parametric down-conversion [7]. 
In this paper we study the behavior of this device as a function of temperature and of the injected current and we discuss the impact of the device's properties on the generated quantum state.

\section{Results}

\subsection{Design and Fabrication}

The challenge in designing such a device lies in simultaneously addressing the electrical injection of the laser and the efficient down-conversion of laser photons in photon pairs.

In the nonlinear process, the energy and momentum of the photons are conserved-nothing is exchanged with the crystal. While energy conservation is straightforward, conserving the momentum requires compensating for the phase velocity mismatch of the three interacting photons, which is usually done by using birefringent crystals. However, even if GaAs present a high second-order nonlinearity, which is beneficial for achieving efficient frequency conversion processes $\left(\chi^{(2)}\right.$ around $110 \mathrm{pm} / \mathrm{V}$ at $1550 \mathrm{~nm}$ [8]), this material lacks natural birefringence. Among different techniques to achieve phase-matching, the recent development of Bragg reflection waveguides $[9,10]$ represents a breakthrough, since this strategy allows us to reduce the total aluminum content (thus avoiding aging problems of the device) and increases the flexibility of effective index engineering. In our case, two Bragg mirrors provide both a photonic band gap vertical confinement for the laser mode (a transverse electric Bragg mode) and total internal reflection claddings for the photon-pairs modes (one $\mathrm{TE}_{00}$ and one $\mathrm{TM}_{00}$ ). The device's design is dictated by trade-offs that must optimize electrical transport, waveguiding and nonlinear interaction at the same time [11]. Figure 1a,b present, respectively, the simulated intensity profile of the laser Bragg mode and the refractive index and doping profiles of the resulting design.

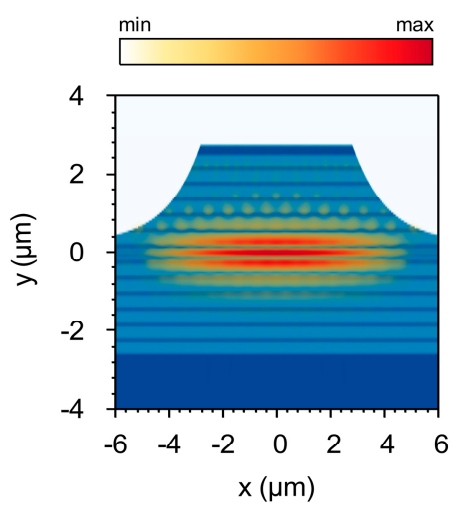

(a)

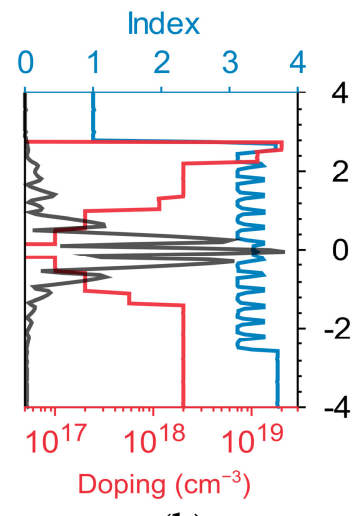

(b)

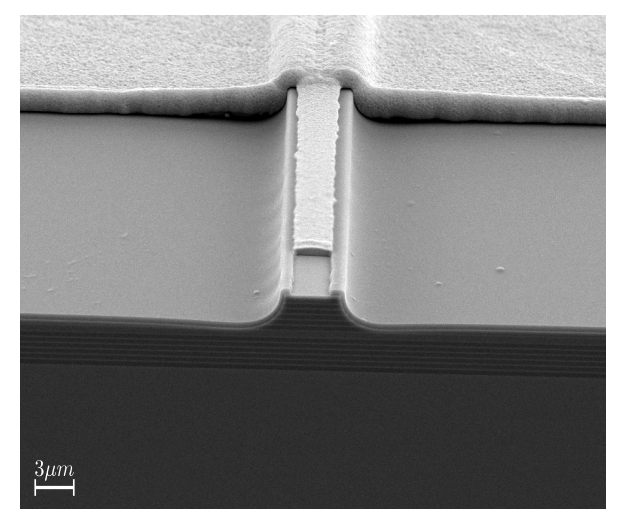

(c)

Figure 1. (a) Simulated intensity profile for the Bragg mode within the waveguide; (b) Refractive index and doping profile of the device; the Bragg mode has a higher overlap with the quantum well than the $\mathrm{TE}_{00}$ mode; (c) SEM image of the sample showing the facet of the sample and the upper electrode with the thick electrolytic layer.

The sample under study has been grown by molecular beam epitaxy on a (100) GaAs substrate. It consists of two $\mathrm{Al}_{0.8} \mathrm{Ga}_{0.2} \mathrm{As} / \mathrm{Al}_{0.25} \mathrm{Ga}_{0.75} \mathrm{As}$ Bragg reflectors of six periods each surrounding a $365 \mathrm{~nm} \mathrm{Al}_{0.45} \mathrm{Ga}_{0.55} \mathrm{As}$ core. A $8.5 \mathrm{~nm} \mathrm{Al}_{0.11} \mathrm{Ga}_{0.89}$ As quantum well is embedded into the core to ensure a gain peaked at $780 \mathrm{~nm}$ (see Figure 1a). The top and bottom Bragg reflectors are, respectively, gradually p-doped and n-doped, from $1 \times 10^{17}$ to $2 \times 10^{18}$, in order to ensure efficient electrical injection of the device. An additional highly doped cap layer is added to protect the device and facilitate electrical injection from the upper contact [12]. The device is processed in a standard waveguide geometry, as presented in Figure 1c: 5-6 $\mu \mathrm{m}$ wide and $2 \mu \mathrm{m}$ deep waveguides are defined by wet etching of the top Bragg reflector. A SiN layer is used for electrical insulation and $\mathrm{Ti} / \mathrm{Au}$ and $\mathrm{Ni} / \mathrm{Ge} / \mathrm{Au} / \mathrm{Ni} / \mathrm{Au}$ 
layer sequences are used for the top and bottom contact, respectively. A thick electrolytic Au layer is used for the top contact, helping heat management. Samples are cleaved into approximately $2 \mathrm{~mm}$ long stripes.

\subsection{Optoelectronic Characterization}

Figure 2a reports the typical power-current-voltage (PIV) curves as a function of the temperature of one of our samples under electrical pumping; the pulse duration is $60 \mathrm{~ns}$ at a repetition rate of $40 \mathrm{kHz}$. These curves attest for the lasing emission. We observe that at $T=291 \mathrm{~K}$, the $\mathrm{I}-\mathrm{V}$ curve shows a resistance of $R=3.56 \Omega$ and a turn-on voltage of $1.83 \mathrm{~V}$, which is very close to the quantum well band gap, thus meaning that no current-blocking effects occur at the hetero-interfaces. The P-I curve displays a threshold current of $I_{t h}=0.274 \mathrm{~A}$, corresponding to threshold density current $J_{t h}=1.5 \pm 0.2 \mathrm{kA} / \mathrm{cm}^{2}$ for a contact surface of $S=1.9( \pm 0.2) \times 10^{-4} \mathrm{~cm}^{2}$.

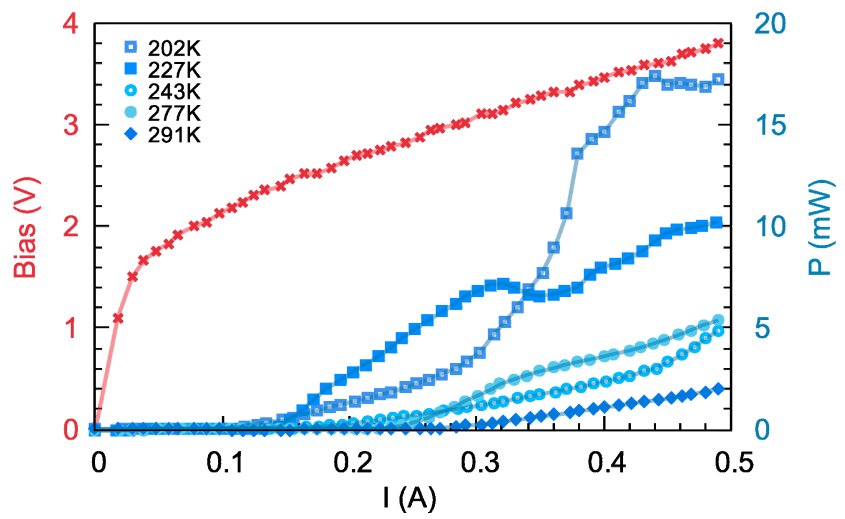

(a)

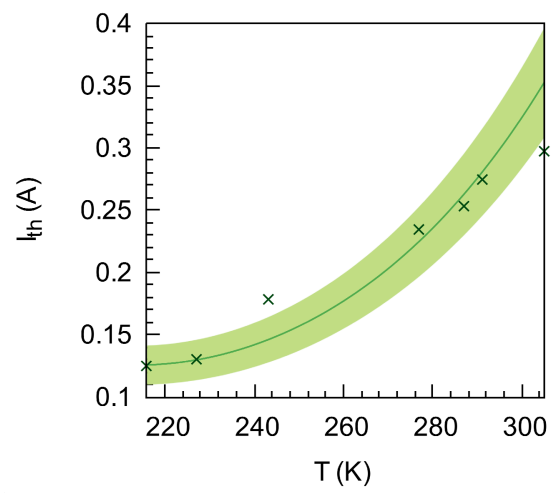

(b)

Figure 2. Laser operation. (a) Voltage bias (crosses) and emitted power versus injected current. The $\mathrm{I}-\mathrm{V}$ curve is measured at $T_{\text {room }}=291 \mathrm{~K}$, while I-P for $\mathrm{T}$ is in the range 202-291 K. At room temperature the laser has a threshold of $I_{t h}=0.274 \mathrm{~A}$, a turn on voltage of $1.8 \mathrm{~V}$; (b) Measured (crosses) and calculated (continuous line) threshold current as a function of temperature. The error band around the calculated threshold corresponds to the uncertainty of the diode surface after the wet etching and cleaving processes.

As the temperature decreases from $T_{\text {room }}=291 \mathrm{~K}$ to $T=202 \mathrm{~K}$, the threshold current decreases exponentially as shown in Figure $2 \mathrm{~b}$, according to $I_{t h} \sim e^{T / T 0}$, with $T_{0}=87.8 \mathrm{~K}$ [13].

These experimental results are compared with the numerical simulations we have developed to design our device; the carrier transport and recombination in the hetero-junction are calculated using a self-consistent solution of the drift-diffusion and Poisson equations. The optical parameters of our modeling are the Bragg mode internal losses, whose value $\alpha=35 \mathrm{~cm}^{-1}$ is extracted from the experimental results obtained by a second-harmonic generation measurement [14]; the overlap integral between the Bragg mode and the quantum well $\Gamma=2.45 \%$, calculated with a commercial eigenmode solver [15]; and the facets modal reflectivity for the Bragg mode $\mathrm{R}=0.86$, calculated by Finite-Difference Time-Domain (FDTD). The radiative recombination time in the quantum well is set to $\tau^{\mathrm{rad}}=5 \times 10^{-17} \mathrm{~m}^{3} \cdot \mathrm{s}^{-1}$, while the Shockley-Read-Hall non-radiative recombination processes is $\tau^{\mathrm{SRH}}=5 \mathrm{~ns}$ [16]. The waveguide length and width are $L=2 \mathrm{~mm}$ and $w=6 \mu \mathrm{m}$, respectively, while the doping profile and aluminum contents correspond to the nominal values of the epitaxial structure.

Figure $2 \mathrm{~b}$ shows the good agreement between the experimental and the calculated threshold current as a function of the temperature in the 216-305 K range, within the uncertainty of the device contact surface $S=1.9( \pm 0.2) \times 10^{-4} \mathrm{~cm}^{2}$.

From the calculated internal resistance of the diode $\left(R_{\text {int }} \approx 1.5 \Omega\right.$ at room temperature) we evaluate the quality of the fabricated contacts, which introduce an additional resistance lower than $R=2.0 \Omega$. 
The spatial intensity distribution of the emitted laser beam above the threshold is studied by imaging the output facet; the recorded near-field and far-field distributions are reported in Figure 3a, showing a clear evidence of emission on the Bragg mode. For higher values of injected current the Bragg mode can be in competition with the fundamental $\mathrm{TE}_{00}$ mode due to index anti-guiding effects coming from a carrier density increase. This might explain the irregular behavior observed in the I-P curve; further studies on this point are in progress.

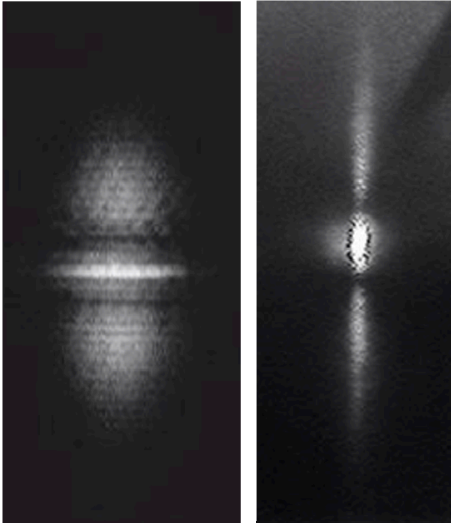

(a)

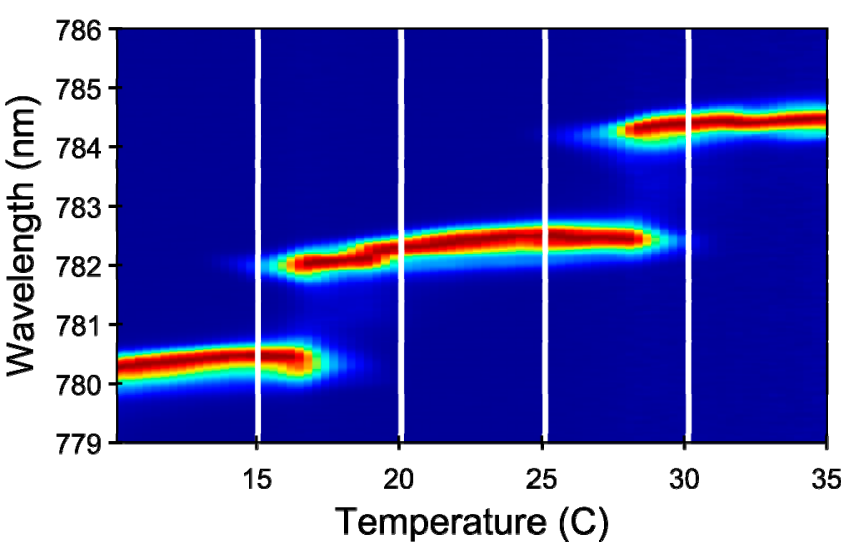

(b)

Figure 3. (a) Measured near- and far-field emissions of a laser emitting on the Bragg mode; (b) Normalized laser emission intensity as a function of wavelength and heat-sink temperature.

Figure $3 \mathrm{~b}$ displays the laser emission intensity spectra as a function of heat-sink temperature, measured with an Optical Spectrum Analyzer for a laser emitting on the Bragg mode at room temperature. The general trend corresponds to the theoretical temperature dependence of the quantum well band gap $\left(0.23 \mathrm{~nm} /{ }^{\circ} \mathrm{C}\right)$; mode hopping is clearly visible with wavelength jumps of approximately $\Delta \lambda \approx 1.5-2 \mathrm{~nm}$.

We notice that these jumps correspond to more than 35 times the spacing between adjacent longitudinal modes of our device; indeed, taking into account the laser length and the modal dispersion, we find $\Delta \lambda_{\mathrm{FSR}}=\lambda^{2} / 2 \mathrm{~L} \times(\mathrm{n}-\lambda \mathrm{dn} / \mathrm{d} \lambda)^{-1} \approx 40 \mathrm{pm}$ [17]. This behavior has already been observed in AlGaAs laser structures [18]; it seems to be explained by the presence of an additional saturable source of losses, resulting from deep-level traps induced in the n-type doped Bragg layers by Si donors. As the traps' absorption is inversely proportional to the photon density, the standing-wave intensity profile of the dominant longitudinal mode $I(z)$ determines the periodicity of the optical loss pattern $\alpha^{\text {traps }}(\mathrm{z})=\alpha_{0} /\left(1+I(z) / I_{s}\right)$ [19]. By integrating along the whole device length, it is possible to show that the dominant longitudinal mode presents smaller losses than the adjacent modes by a factor $\Delta \alpha \approx 0.05-0.1 \alpha_{0}$. In order to verify that our observations are related to this physical process, we have estimated $\Delta \alpha$, calculating the overlaps of the Bragg mode with each n-doped layer. By assuming the reflectivity $\mathrm{R}=0.86$, we find $\alpha_{0}=5.54 \mathrm{~cm}^{-1}$ at room temperature leading to $\Delta \alpha \approx 0.28-0.55 \mathrm{~cm}^{-1}$, which is compatible with our experimental results (Figure $3 b$ ).

The demonstration of the photon pair emission in the telecom range has been done by performing time correlation measurements under electrical injection in a pulsed regime at $601 \mathrm{~mA}$. The emerging TE and TM photon pairs, corresponding to a type-II SPDC, are detected with two InGaAs single-photon avalanche photodiodes (IdQ201) having 20\% detection efficiency and a $50 \mathrm{~ns}$ gate, synchronized with the current pulses. A time-to-digital converter is used to analyze the time correlations between cross-polarized photons, separated by a polarization beam splitter. Figure 4 shows a histogram of the detection time delays at $T=25^{\circ} \mathrm{C}$. The sharp peak emerging from the background is the evidence that photons are produced by pairs. From these data, taking into account the optical losses along the optical path, we estimate an internal generation efficiency of the device of $\sim 10^{-10}$ pairs/injected electron. 


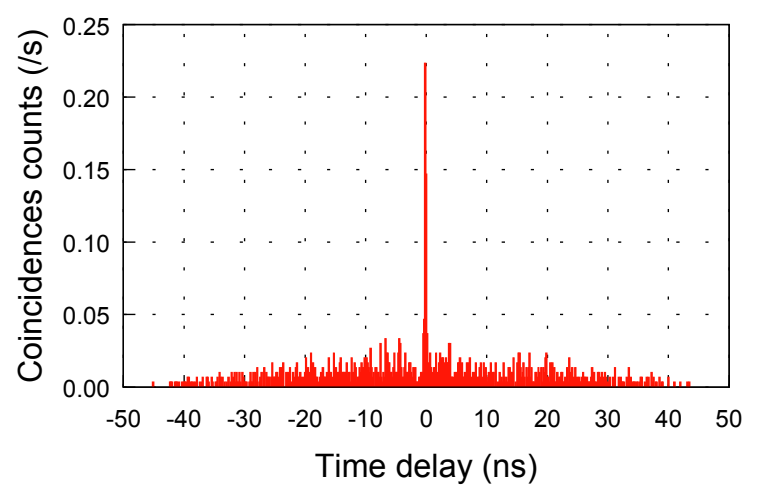

Figure 4. Time-correlation histogram of TE/TM photons around $1.57 \mu \mathrm{m}$ at $T=15^{\circ} \mathrm{C}$. This sample is electrically injected with current pulses of $601 \mathrm{~mA}$, a duration of $60 \mathrm{~ns}$ and a repetition rate of $40 \mathrm{kHz}$. The data were accumulated during $600 \mathrm{~s}$ with a sampling resolution of $162 \mathrm{ps}$.

\section{Discussion and Conclusions}

In this section we discuss the impact of the performances of our devices on the quality of the quantum state of the emitted photons and we give some perspectives.

The signal-to-noise ratio (SNR) is evaluated by taking the number of true coincidences within the Full Width Half Maximum (FWHM) of the peak over the background signal on the same time window; Figure 5 reports the SNR value as a function of the injected current for the same sample characterized in Figure 4. For a current value below the laser threshold, no "true coincidence" peak emerges from the noise. For a current value between $450 \mathrm{~mA}$ and $600 \mathrm{~mA}$, the SNR increases linearly with the injected current as expected from the typical diode behavior of the device. At high current values the SNR saturates, probably due to thermal effects within the device having an impact on the laser operation and the phase-matching curve.

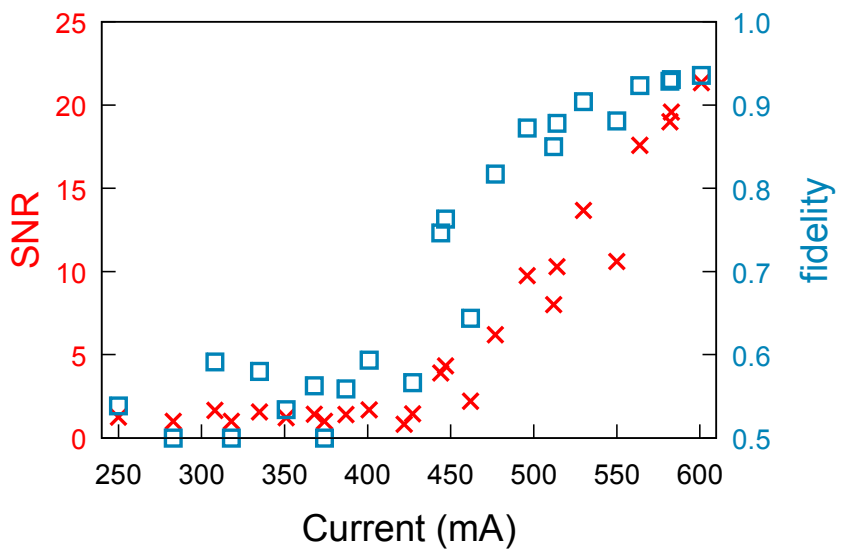

Figure 5. Signal-to-noise ratio and fidelity to a Bell state as a function of the current for one of our devices.

Assuming that the source emits a Werner state, we can estimate the fidelity $\mathrm{F}$ to the maximally entangled Bell state $\left|\psi_{+}\right\rangle: \mathrm{F}=(1+3 \mathrm{P}) / 4$ with $\mathrm{P}=\mathrm{SNR} /(2+\mathrm{SNR})$. The value of $\mathrm{F}$ as a function of the current is also given in Figure 5: we observe that for an injected current of $600 \mathrm{~mA}$, F should reach the value of $93 \%$, which is compliant with an experimental violation of Bell's inequality.

Recently, we have improved the fabrication method of our devices, passing from wet to dry etching to define the waveguides; this has allowed increasing the confinement and overlap of the interacting modes (and consequently the nonlinear process efficiency) while keeping a low level of 
optical losses. We have thus reached a SNR value of 1730 , which potentially would bring us to the condition of achieving a fidelity value of $99 \%$ [20].

In order to check the possibility to drive the device in continuous wave (CW) operation, we have measured the output power as a function of the duty cycle: the observation of a constant peak output power for a duty cycle up to $66 \%$ indicates that a CW operation is possible [12].

In parallel, quantum optics experiments on the photon pairs generated by passive samples based on the same phase-matching technique have allowed us to demonstrate high values of indistinguishability [21,22] and entanglement on polarization [23] and energy-time [22]. The quality of the produced quantum state as well as its compatibility with the telecom network have also allowed to use these sources in multi-user quantum key distribution protocols using standard dense wavelength division multiplexers [24]. This approach seems also to be promising for the control of the frequency correlations of the emitted biphoton state; for example, original designs of $\mathrm{Al}_{\mathrm{x}} \mathrm{Ga}_{1-\mathrm{x}} \mathrm{N}$ devices have been proposed in which quasi-phase-matching of the waveguide core is used to achieve phase-matching at the desired wavelength, while the control of waveguide dispersion is used to control the frequency correlation between the generated photons [25].

In conclusion, Bragg reflection waveguides emerge as an extremely attractive and versatile platform on which to perform scalable photonics-based quantum information tasks. Their ability to be electrically driven at room temperature lets us envision the use of III-V semiconductors for a widespread diffusion of quantum technologies.

Acknowledgments: This work has been funded by the Agence Nationale de la Recherche (ANR-14-CE26-0029-01); the Direction Générale de l'Armement (DGA); the French RENATECH; the Institut Universitaire de France. The authors thank A. Orieux for the design of the sample.

Author Contributions: Claire Autebert, Yacine Halioua, Fabien Boitier did the experiments, Giorgio Maltese, Maria Amanti, Carlo Sirtori contributed to the numerical simulations and interpretation, Aristide Lemaitre did the sample growth; Sara Ducci supervised all the stages of the work and wrote the paper.

Conflicts of Interest: The authors declare no conflict of interest.

\section{References}

1. Grassani, D.; Azzini, S.; Liscidini, M.; Galli, M.; Strain, M.J.; Sorel, M.; Sipe, J.E.; Bajoni, D. MicRometer-scale integrated silicon source of time-energy entangled photons. Optica 2015, 2, 88-94. [CrossRef]

2. Matthews, J.C.F.; Politi, A.; Stefanov, A.; O’Brien, J.L. Manipulation of multiphoton entanglement in waveguide quantum circuits. Nat. Photonics 2009, 3, 346-350. [CrossRef]

3. Spagnolo, N.; Vitelli, C.; Bentivegna, M.; Brod, D.J.; Crespi, A.; Flamini, F.; Giacomini, S.; Milani, G.; Ramponi, R.; Mataloni, P.; et al. Experimental validation of photonic boson sampling. Nat. Photonics 2014, 8, 615-620. [CrossRef]

4. Spring, J.B.; Metcalf, B.J.; Humphreys, P.C.; Kolthammer, W.S.; Jin, X.M.; Barbieri, M.; Datta, A.; Thomas-Peter, N.; Langford, N.K.; Kundys, D.; et al. Boson sampling on a photonic chip. Science 2013, 339, 798-799. [CrossRef] [PubMed]

5. Sprengers, J.P.; Gaggero, A.; Sahin, D.; Jahanmirinejad, S.; Frucci, G.; Mattioli, F.; Leoni, R.; Beetz, J.; Lermer, M.; Kamp, M.; et al. Waveguide superconducting single-photon detectors for integrated quantum photonic circuits. Appl. Phys. Lett. 2011, 99, 181110. [CrossRef]

6. Somaschi, N.; Giesz, V.; de Santis, L.; Loredo, J.C.; Almeida, M.P.; Hornecker, G.; Portalupi, S.L.; Grange, T.; Antón, C.; Demory, J.; et al. Near-optimal single-photon sources in the solid state. Nat. Photonics 2016, 10, 340-345. [CrossRef]

7. Boitier, F.; Orieux, A.; Autebert, C.; Lemaitre, A.; Galopin, E.; Manquest, C.; Sirtori, C.; Favero, I.; Leo, G.; Ducci, S. Electrically injected photon-pair source at room temperature. Phys. Rev. Lett. 2014, 112, 183901. [CrossRef] [PubMed]

8. Shoji, I.; Kondo, T.; Ito, R. Second-order nonlinear susceptibilities of various dielectric and semiconductor materials. Opt. Quantum Electron. 2002, 34, 797-833. [CrossRef]

9. Yeh, P.; Yariv, A. Bragg reflection waveguides. Opt. Commun. 1976, 19, 427-430. [CrossRef] 
10. Helmy, A.; Bijlani, B.; Abolghasem, P. Phase matching in monolithic Bragg reflection waveguides. Opt. Lett. 2007, 32, 2399-2401. [CrossRef] [PubMed]

11. Rossi, A.D.; Ortiz, V.; Calligaro, M.; Vinter, B.; Nagle, J.; Ducci, S.; Berger, V. A third-order-mode laser diode for quantum communication. Semicond. Sci. Technol. 2004, 19, L99-L102. [CrossRef]

12. Autebert, C.; Boucher, G.; Boitier, F.; Eckstein, A.; Favero, I.; Leo, G.; Ducci, S. Photon pair sources in algaas: From electrical injection to quantum state engineering. J. Mod. Opt. 2015, 62, 1739-1745. [CrossRef]

13. Svelto, O.; Hanna, D.C. Principles of Lasers, 5th ed.; Springer: Berlin, Gemany, 2012.

14. Ravaro, M.; Guillotel, E.; Le Dû, M.; Manquest, C.; Marcadet, X.; Ducci, S.; Berger, V.; Leo, G. Nonlinear measurement of mid-infrared absorption in AlOx waveguides. Appl. Phy. Lett. 2008, 92, 151111. [CrossRef]

15. Lumerical Solutions, I. Available online: http://www.lumerical.com/tcad-products/fdtd (accessed on 25 June 2016).

16. Marvin, D.C.; Moss, S.C.; Halle, L.F. Analysis of transient photoluminescence measurements on GaAs and AlGaAs double heterostructures. J. Appl. Phys. 1992, 72, 1970-1984. [CrossRef]

17. Lanco, L. Sources Semiconductrices de Photons Jumeaux. Ph.D. Thesis, Université Paris Diderot, Paris, France, 2006.

18. Chinone, N.; Kuroda, T.; Ohtoshi, T.; Takahashi, T.; Kajimura, T. Mode-hopping noise in index-guided semiconductor lasers and its reduction by saturable absorbers. IEEE J. Quantum Electron. 1985, 21, 1264-1270. [CrossRef]

19. Copeland, J. Single-mode stabilization by traps in semiconductor lasers. IEEE J. Quantum Electron. 1980, 16, 721-727. [CrossRef]

20. Autebert, C.; Lemaître, A.; Ducci, S. Highly efficient parametric down conversion in a monolithic AlGaAs waveguide. 2016, in preparation.

21. Günthner, T.; Pressl, B.; Laiho, K.; Geßler, J.; Höfling, S.; Kamp, M.; Schneider, C.; Weihs, G. Broadband indistinguishability from bright parametric downconversion in a semiconductor waveguide. J. Opt. 2015, 17, 125201. [CrossRef]

22. Autebert, C.; Bruno, N.; Martin, A.; Lemaitre, A.; Carbonell, C.G.; Favero, I.; Leo, G.; Zbinden, H.; Ducci, S. Integrated algaas source of highly indistinguishable and energy-time entangled photons. Optica 2016, 3 , 143-146. [CrossRef]

23. Horn, R.T.; Kolenderski, P.; Kang, D.; Abolghasem, P.; Scarcella, C.; Della Frera, A.; Tosi, A.; Helt, L.G.; Zhukovsky, S.V.; Sipe, J.E.; et al. Inherent polarization entanglement generated from a monolithic semiconductor chip. Sci. Rep. 2013, 3, 2314. [CrossRef] [PubMed]

24. Autebert, C.; Trapateau, J.; Orieux, A.; Lemaitre, A.; Carbonell, C.G.; Diamanti, E.; Zaquine, I.; Ducci, S. Multi-User Quantum Key Distribution with Entangled Photons from an AlGaAs Chip. 2016. Available online: http:/ / arxiv.org/abs/1607.01693 (accessed on 6 July 2016).

25. Svozilyk, J.; Hendrych, M.; Helmy, A.S.; Torres, J.P. Generation of paired photons in a quantum separable state in Bragg reflection waveguides. Opt. Express 2011, 19, 3115-3123. [CrossRef] [PubMed]

(C) 2016 by the authors; licensee MDPI, Basel, Switzerland. This article is an open access article distributed under the terms and conditions of the Creative Commons Attribution (CC-BY) license (http://creativecommons.org/licenses/by/4.0/). 